Technological University Dublin

DƯBLIN

ARROW@TU Dublin

2009-01-01

\title{
Use of a Single-Multiple-Single-Mode Fiber Filter for Interrogating Fiber Bragg Grating Strain Sensors with Dynamic Temperature Compensation
}

\author{
Qiang wu \\ Technological University Dublin, qiang.wu@tudublin.ie \\ Muhammad Hatta \\ Technological University Dublin \\ Yuliya Semenova \\ Technological University Dublin, yuliya.semenova@tudublin.ie
}

See next page for additional authors

Follow this and additional works at: https://arrow.tudublin.ie/engscheceart

Part of the Electrical and Computer Engineering Commons

\section{Recommended Citation}

Wu, Q. et al (2009) Use of a Single-Multiple-Single-Mode Fiber Filter for Interrogating Fiber Bragg Grating Strain Sensors with Dynamic Temperature Compensation. Applied Optics, Vol.48, no. 29, p.5451. doi: 10.1364/AO.48.005451

This Article is brought to you for free and open access by the School of Electrical and Electronic Engineering at ARROW@TU Dublin. It has been accepted for inclusion in Articles by an authorized administrator of ARROW@TU Dublin. For more information, please contact arrow.admin@tudublin.ie, aisling.coyne@tudublin.ie, gerard.connolly@tudublin.ie.

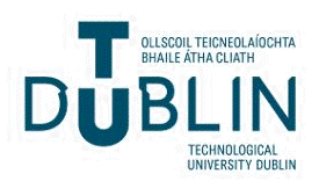


Authors

Qiang wu, Muhammad Hatta, Yuliya Semenova, and Gerald Farrell

This article is available at ARROW@TU Dublin: https://arrow.tudublin.ie/engscheceart/92 


\title{
Use of a single-multiple-single-mode fiber filter for interrogating fiber Bragg grating strain sensors with dynamic temperature compensation
}

\author{
Qiang Wu, ${ }^{\star}$ Agus Muhammad Hatta, Yuliya Semenova, and Gerald Farrell \\ Photonics Research Center, School of Electronic and Communications Engineering, \\ Dublin Institute of Technology, Kevin Street, Dublin 8, Ireland \\ ${ }^{*}$ Corresponding author: qiang.wu@ dit.ie
}

Received 15 June 2009; revised 10 September 2009; accepted 10 September 2009; posted 11 September 2009 (Doc. ID 112781); published 1 October 2009

\begin{abstract}
An interrogation technique for fiber Bragg grating (FBG) strain sensors with dynamic temperature compensation using a single-multiple-single-mode (SMS) fiber filter as a temperature compensating element is presented. Experimental results show that this technique offers a resolution of better than $3.4 \mu \varepsilon$ for strain measurements in the range from 0 to $1667 \mu \varepsilon$, and the temperature induced error is as low as $34 \mu \varepsilon$ in the temperature range from 10 to $60^{\circ} \mathrm{C}$. The temperature induced error could be further reduced if the temperature sensitivity (the rate of temperature induced wavelength shift) of the SMS filter was closer to that of the FBG sensor. This can be achieved by selecting a multimode fiber for the SMS filter with appropriate parameters. The proposed technique can be modified for simultaneous measurements of strain and temperature with an experimentally achieved resolution of better than $1^{\circ} \mathrm{C}$. (C) 2009 Optical Society of America

OCIS codes: $\quad 060.2370,060.3735$.
\end{abstract}

\section{Introduction}

Fiber Bragg grating (FBG) based optical sensing is widely used for monitoring bridges and other civil structures [1], river surveillance, and aerospace structural health monitoring [2]. The cross sensitivity of FBGs to temperature and strain means that it is necessary to discriminate between these two measurands, as in most applications the sensors operate in a randomly changing temperature environment. Changes to the environmental temperature will lead to a shift of the FBG center wavelength and hence will result in an erroneous strain measurement if the influence of temperature is not accounted for. Numerous techniques have been proposed to discriminate between the influence of temperature and strain. In 1997, Brady et al. proposed using the firstand second-order diffraction wavelengths of an FBG to realize simultaneous measurements of tempera-

0003-6935/09/295451-08 $\$ 15.00 / 0$

(C) 2009 Optical Society of America ture and strain [3]. In 1998, Haran et al. used an additional FBG sensor in the same environment to subtract the temperature information and hence allow the strain information to be accurately determined [4]. In 2006, Jin et al. used an encapsulated FBG in a tapered polymer to measure both temperature and stress via a matrix of coefficients by measuring both the chirp and central wavelength shift [5], and in 2007, Shao et al. presented a high-resolution strain and temperature sensor based on a polarimetric distributed Bragg reflector fiber laser [6]. More recently, Nguyen et al. proposed using a Lyot fiber filter combined with an FBG to measure both temperature and strain via a coefficient matrix [7]. All these techniques employed an optical spectrum analyzer (OSA) to extract either wavelength or bandwidth information, which significantly increases the cost of the system and limits scanning speed. As an alterative to an OSA, ratiometric wavelength measurement is a simple, high speed, and cost effective scheme $[8,9]$. Techniques for temperature compensating for ratiometric systems were also extensively 
investigated. Miao et al. used a tilted FBG to realize dynamic temperature compensation [10]. This dynamic temperature compensation technique is simple, straightforward, and especially attractive when the temperature information is not needed and only the strain is of interest. Recently Zhou et al. used a single-multiple-single-mode (SMS) fiber filter to realize a simultaneous measurement of both temperature and strain by using an experimentally determined matrix of strain and temperature coefficients [11]. In this technique the SMS filter is used for simultaneous measurement of temperature and strain offering a more cost effective solution. The disadvantage of such an approach is that the temperature and strain coefficient matrix must be previously determined, and therefore both temperature and strain must be measured independently in order to experimentally find the values of the matrix coefficients. Since the SMS filter used in [11] had different temperature sensitivity $\left(-55 \mathrm{pm} /{ }^{\circ} \mathrm{C}\right)$ compared to that of an FBG (typically $10 \mathrm{pm} /{ }^{\circ} \mathrm{C}$ ), it is impossible to employ such an SMS filter for dynamic temperature compensation without the use of a temperature and strain coefficient matrix. However, if it can be arranged that both SMS filter and FBG have similar temperature sensitivity, dynamic temperature compensation is much simpler.

In this paper we propose a dynamic temperature compensating technique for FBG sensing based on combining an FBG sensor with an SMS edge filter in a ratiometric measurement system. The system description and analysis are provided in Section 2. A detailed design for a suitable SMS edge filter with a temperature sensitivity of $10 \mathrm{pm} /{ }^{\circ} \mathrm{C}$ is given in $\mathrm{Sec}$ tion 3. Finally, the results of experimental verification and further investigations for simultaneous measurement of both strain and temperature by using different system configurations are provided in Sections $\underline{4}$ and $\underline{5}$.

\section{System Description and Analysis}

A schematic diagram of a dynamic temperature compensating interrogation system using an SMS edge filter is shown in Fig. 1. In Fig. 1 the FBG acts as a strain sensor, and the SMS fiber filter acts as an edge filter that is used to determine the shift of the FBG wavelength. Both the FBG and SMS filter are exposed to the same temperature conditions. The signal from a broadband optical source is reflected from the FBG and is then split into two paths: one

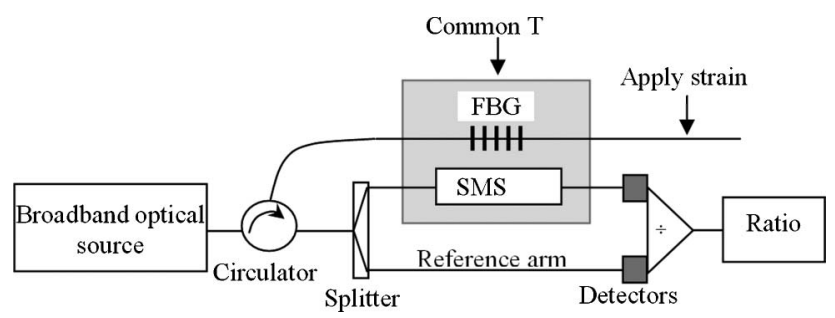

Fig. 1. Schematic diagram of the temperature compensating ratiometric interrogation system. part of the signal passes the SMS filter and is detected by a photodiode acting as a signal arm, and the other is directly supplied to the second photodiode acting as a reference arm. At a constant temperature the FBG wavelength shifts as strain changes, and hence the output ratio varies. However, if both temperature and strain change, a single ratio measurement is not sufficient to discriminate between the two parameters. In a special case when both the edge filter and the FBG sensor have the same temperature sensitivity, a change in temperature produces similar shifts of the filter spectrum and the Bragg wavelength, so the measured ratio will remain unchanged at a fixed strain and will only vary as a result of strain changes. The principle can be explained with the help of Fig. 2 .

In Fig. 2, as temperature changes from 20 to $60^{\circ} \mathrm{C}$, both the FBG and the SMS filter spectra experience similar shifts along the wavelength axis, and hence the relative wavelength shift between them is very small. In this case the level of optical power reflected from the FBG and passed through the SMS filter should remain the same, and hence the measured ratio will remain constant when temperature change occurs. On the other hand, a change in strain applied to the FBG will result in a relative wavelength shift between the FBG and the SMS filter spectra. In this case the optical power level reflected from the FBG and passed through the edge filter will change, and hence a change in the measured ratio will be detected by the photodiodes. The strain information can thus be extracted by monitoring the ratio variations.

\section{Design of Single-Multiple-Single-Mode Filter}

The schematic diagram of an SMS fiber structure is shown in Fig. 3. The multimode fiber (MMF) in Fig. 3 has a step-index profile. As described in [12], the SMS fiber structure can be designed to act as bandpass filter such that one edge of the bandpass response is used to implement an edge filter in a ratiometric measurement scheme.

The temperature sensitivity of $-55 \mathrm{pm} /{ }^{\circ} \mathrm{C}$ for an SMS fiber edge filter was previously reported in [11]. The temperature sensitivity of such SMS filters

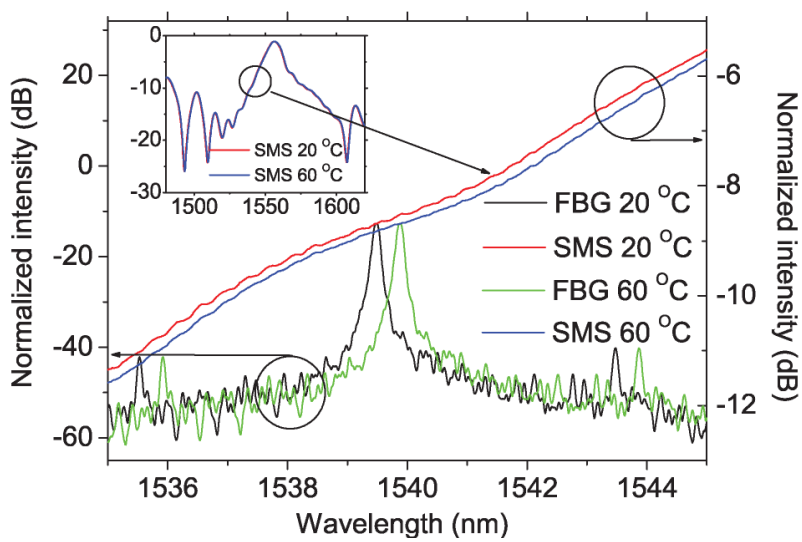

Fig. 2. (Color online) Spectra response of a FBG and a SMS filter at temperatures of $20^{\circ} \mathrm{C}$ and $60^{\circ} \mathrm{C}$. 


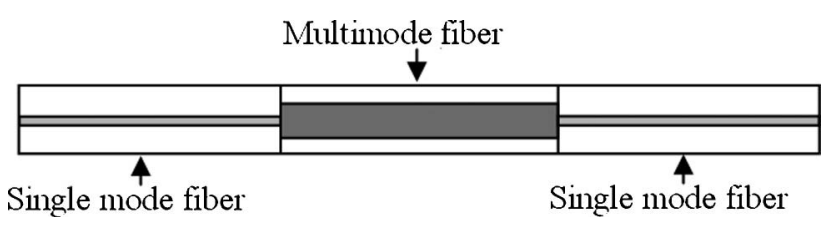

Fig. 3. Schematic diagram of an SMS fiber structure.

depends on parameters of the MMF section. Our aim is to design an SMS fiber edge filter that has temperature sensitivity similar to that of a typical FBG sensor $\left(\sim 10 \mathrm{pm} /{ }^{\circ} \mathrm{C}\right)$.

To design a SMS fiber filter, we used modal propagation analysis (MPA) for linearly polarized (LP) modes $[12,13]$. In the MPA, the input light is assumed to have the fundamental mode field distribution $E(r, 0)$ of the single mode fiber (SMF). The input field can be decomposed into the eigenmodes $\left\{L P_{n m}\right\}$ of the MMF when the light enters the MMF section. Only the $L P_{0 \nu}$ modes of the MMF will be excited due to the circular symmetry of the input field and assuming ideal alignment of the fiber axes of the SMF and the MMF $[12,13]$.

Defining the field profile of $L P_{0 \nu}$ as $F_{\nu}(r)$ (the eigenmodes of the MMF are normalized as $\int_{0}^{\infty}|E(r, 0)|^{2} r \mathrm{~d} r=\int_{0}^{\infty}\left|F_{v}(r)\right|^{2} r \mathrm{~d} r, \quad \nu=1,2,3, \ldots m$, where $m$ is the number of modes in the MMF), the input field at the MMF can be written as
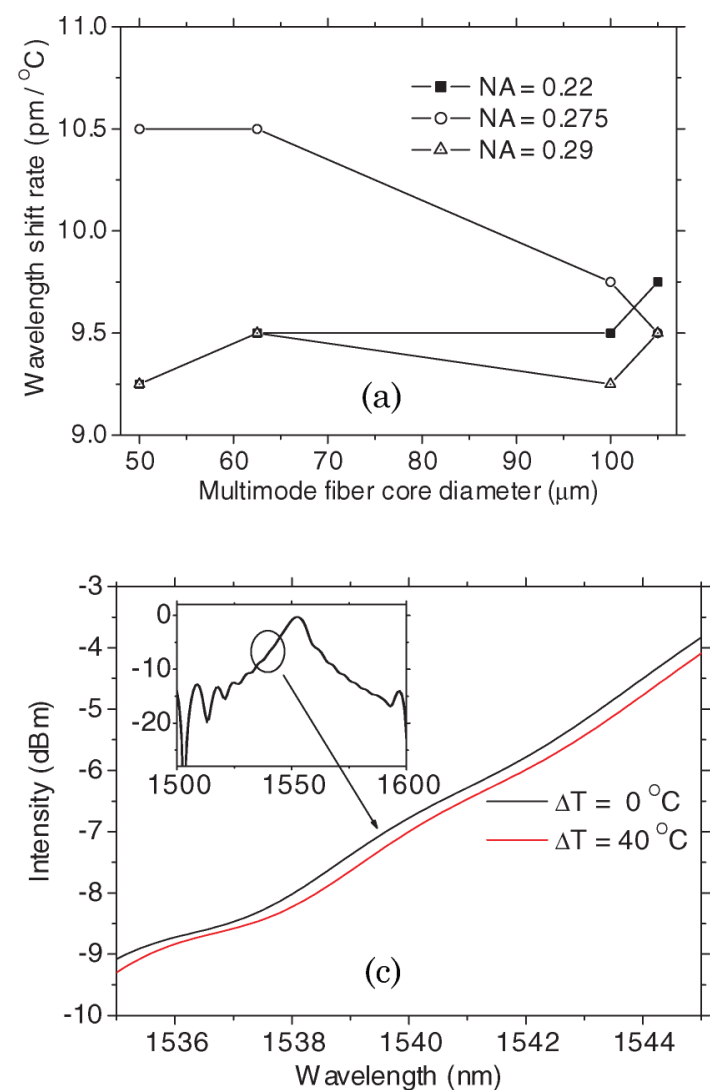

$$
E(r, 0)=\sum_{\nu=1}^{m} c_{\nu} F_{\nu}(r)
$$

where $c_{\nu}$ is the excitation coefficient of each mode. The coefficient $c_{\nu}$ can be calculated by an overlap integral between $E(r, 0)$ and $F_{\nu}(r)$ :

$$
c_{\nu}=\frac{\int_{0}^{\infty} E(r, 0) F_{\nu}(r) r \mathrm{~d} r}{\int_{0}^{\infty} F_{\nu}(r, 0) F_{\nu}(r) r \mathrm{~d} r} .
$$

As the light propagates in the MMF section, the field at a propagation distance $z$ can be calculated by

$$
E(r, z)=\sum_{\nu=1}^{m} c_{\nu} F_{\nu}(r) \exp \left(j \beta_{\nu} z\right)
$$

where $\beta_{\nu}$ is the propagation constant of each eigenmode of the MMF. The transmission loss in decibels can be calculated by using the overlap integral method between $E(r, z)$ and the eigenmode of the output $\mathrm{SMF} E_{0}(r)$ as

$$
L_{s}(z)=10 \cdot \log _{10}\left(\frac{\left|\int_{0}^{\infty} E(r, z) E_{0}(r) r \mathrm{~d} r\right|^{2}}{\int_{0}^{\infty}|E(r, z)|^{2} r \mathrm{~d} r \int_{0}^{\infty}\left|E_{0}(r)\right|^{2} r \mathrm{~d} r}\right) .
$$
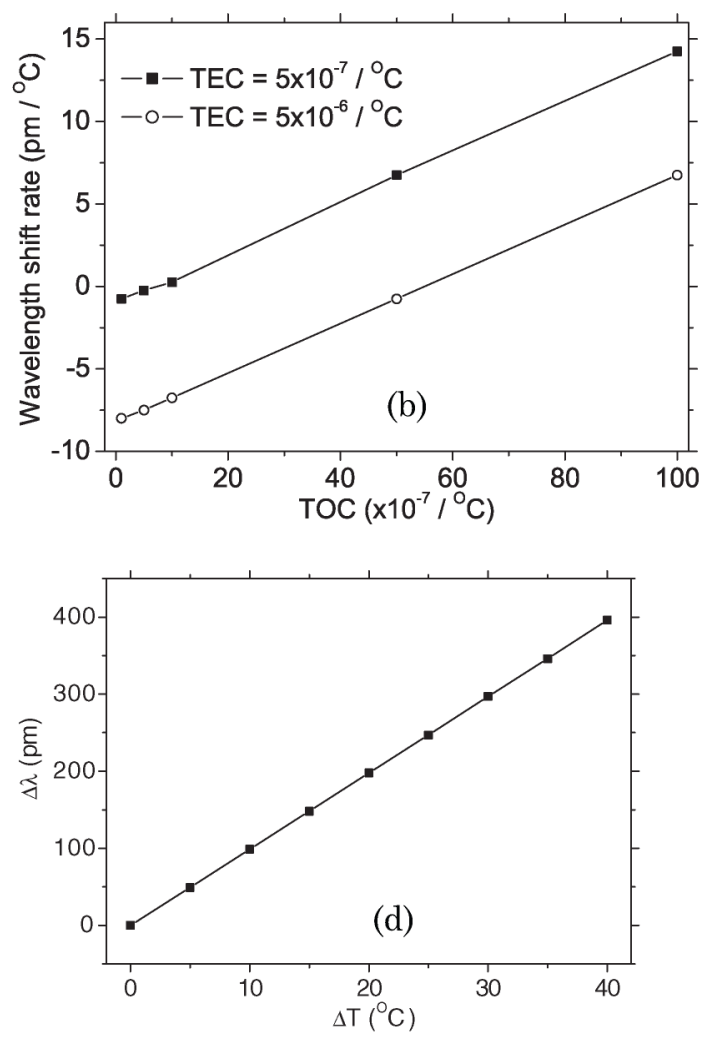

Fig. 4. (Color online) Wavelength shift rate for different (a) MMF core diameter and NA and (b) TEC and TOC; (c) calculated spectral response of an SMS filter; (d) wavelength shift versus temperature. 


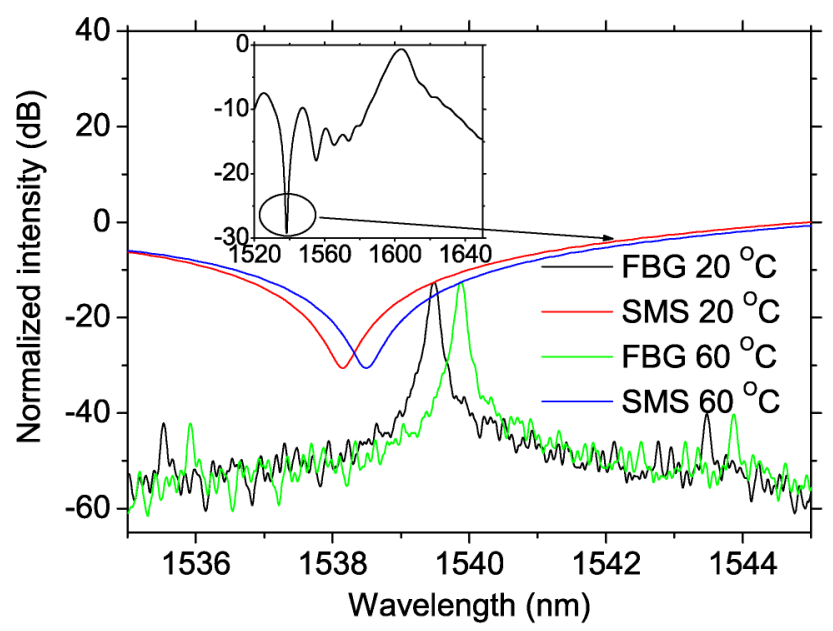

Fig. 5. (Color online) Spectral responses of the FBG and the SMS filter at temperatures of $20^{\circ} \mathrm{C}$ and $60^{\circ} \mathrm{C}$.

It was shown previously $[12,13]$ that, at a reimaging distance, the SMS filter is highly wavelength dependent and operates as a bandpass filter. For the purpose of designing an edge filter, the bandpass response can be considered as two edge responses at each side of the central wavelength. Consequently the device can behave as an edge filter for a selected wavelength range [14].

It is well known that there are two parameters that characterize the effect of temperature on the fiber: the thermal expansion coefficient (TEC) and the thermo-optic coefficient (TOC). The TEC characterizes the physical expansion or contraction of the material's volume, while the TOC characterizes refractive index change in response to a change of temperature. Using the TEC and TOC, the change in core radius $(R), \mathrm{MMF}$ length $(L)$, and refractive index $(n)$ due to a temperature variation $(\Delta T)$ can be expressed, respectively, as

$$
\begin{aligned}
R_{(\mathrm{smf}, \mathrm{mmf})_{T}}= & R_{(\mathrm{smf}, \mathrm{mmf})_{0}}+\alpha \cdot R_{(\mathrm{smf}, \mathrm{mmf})_{0}} \cdot \Delta T, \\
& L_{T}=L_{0}+\alpha \cdot L_{0} \cdot \Delta T, \\
n_{(\text {core }, \text { clad })_{T}}= & n_{(\text {core, }, \text { clad })_{0}}+\xi \cdot n_{(\text {core, clad })_{0}} \cdot \Delta T,
\end{aligned}
$$

where $\alpha$ and $\xi$ are the TEC and the TOC, respectively.

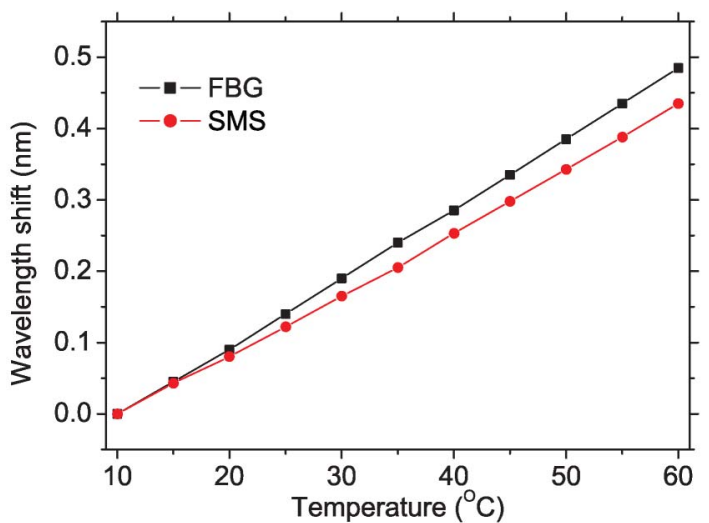

Fig. 6. (Color online) Measured wavelength shifts versus temperature.

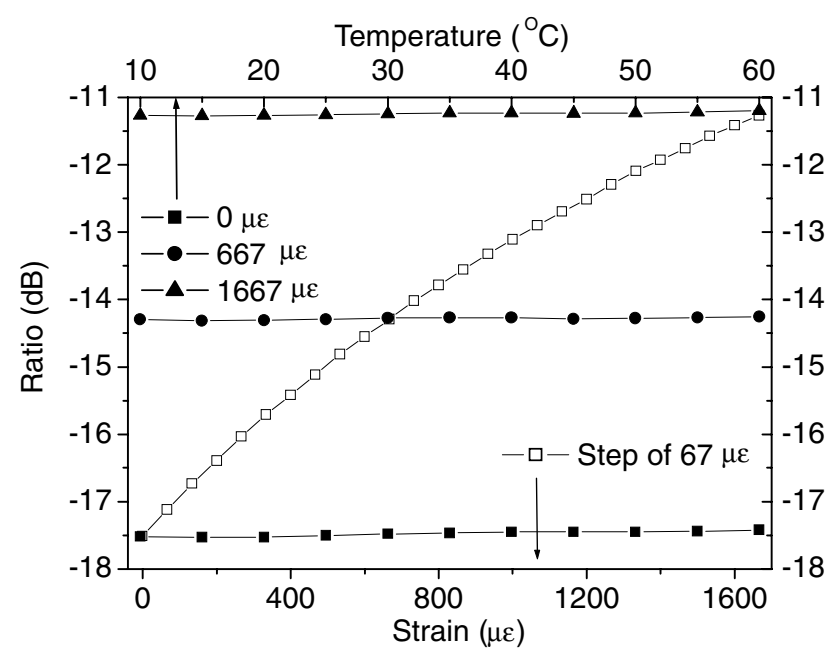

Fig. 7. Measured ratio versus strain at room temperature (bottom axis) and measured ratio versus temperature at fixed strain (top axis).

First, the influence of the MMF core diameter and numerical aperture (NA) on the temperature sensitivity of the filter's response were investigated as shown in Fig. 4(a). In this simulation, the value of the coefficients TEC and TOC were assumed to be $\alpha=5 \times 10^{-7} /{ }^{\circ} \mathrm{C}$ and $\xi=6.9 \times 10^{-6} /{ }^{\circ} \mathrm{C}$ for both the $\mathrm{SMF}$ and the MMF [15]. Other parameters used in our simulations are the following: the refractive indices of the SMF core and cladding are 1.4504 and 1.4447, respectively; the core diameter is $8.3 \mu \mathrm{m}$; the refractive index of the MMF core is 1.4446, and the MMF length is $42.82 \mathrm{~mm}$.

From Fig. 4(a) it can be seen that as either MMF NA or core diameter changes, the rate of temperature induced shift of the filter spectrum also changes, but the variation is quite small, especially for larger core diameters $(>100 \mu \mathrm{m})$. This indicates that both NA and core diameter of the MMF have limited influence on the temperature sensitivity of the SMS edge filter.

Further simulations were carried out to determine the influence of TEC and TOC on the SMS filter wavelength shift rate. Figure 4(b) shows simulation results for the wavelength shift rate versus TOC for two different TEC values for a MMF with $\mathrm{NA}=0.22$ and core diameter of $105 \mu \mathrm{m}$. From this figure one can see that as the TOC increases, the wavelength shift rate also increases significantly in a linear fashion. A negative wavelength shift rate can be obtained at a lower TOC and a higher TEC, which is a possible reason for the result of $-55 \mathrm{pm} /{ }^{\circ} \mathrm{C}$ in [11]. Figure 4(b) also shows that in order to achieve a wavelength shift rate around $10 \mathrm{pm} /{ }^{\circ} \mathrm{C}$ for the SMS filter, a MMF with $\mathrm{NA}=0.22, \alpha=5 \times 10^{-7} /{ }^{\circ} \mathrm{C}$, and $\xi=6.9 \times 10^{-6} /{ }^{\circ} \mathrm{C}$ is a possible option.

The simulated transmission spectrum of the relevant portion of an SMS edge filter with the above parameters is shown in Fig. 4(c) at room temperature $20^{\circ} \mathrm{C}\left(\Delta T=0{ }^{\circ} \mathrm{C}\right)$ and after a $\Delta T=40^{\circ} \mathrm{C}$ increase.

The wavelength shift of the filter spectrum versus temperature change for the SMS filter with the 

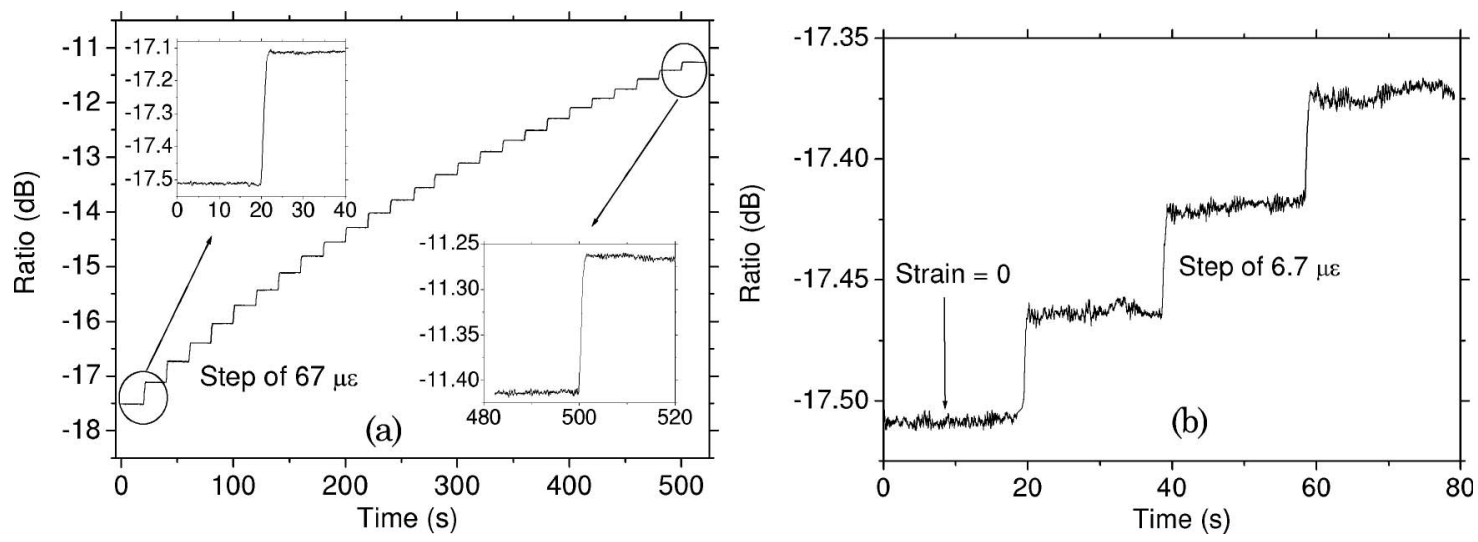

Fig. 8. Measured ratio variations versus time with step change of (a) $67 \mu \varepsilon$ from 0 to $1667 \mu \varepsilon$ and (b) $6.7 \mu \varepsilon$ from 0 to $20 \mu \varepsilon$.

above MMF parameters is shown in Fig. 4(d). It can be seen that for such an SMS filter the spectral response shifts toward longer wavelengths as the temperature increases. The wavelength shift is a linear function of the temperature change, and the temperature sensitivity can be determined as $9.9 \mathrm{pm} /{ }^{\circ} \mathrm{C}$, which is very close to that of a typical FBG.

\section{Experimental Verification}

Experimental investigations were carried out for the proposed system based on a typical FBG sensor and an SMS fiber filter designed based on our simulations. The single-mode and multimode fibers used in our experiments were SMF28 and AFS105/125Y, respectively. The chosen MMF has a refractive index of the core equal to 1.4446 , core radius of $52.5 \mu \mathrm{m}$, and $\mathrm{NA}=0.22$. The MMF section has a length of $40.1 \mathrm{~mm}$.

The spectral responses of the SMS filter and the FBG are shown in Fig. 5.

First, the wavelength shifts of the FBG and SMS filter spectra versus temperature were measured using an OSA, and the results are shown in Fig. 6 .

From Fig. 6 it can be determined that the FBG and the SMS filter have temperature sensitivities of $9.7 \mathrm{pm} /{ }^{\circ} \mathrm{C}$ and $8.7 \mathrm{pm} /{ }^{\circ} \mathrm{C}$ respectively, while the maximum relative wavelength shift between the FBG and SMS filter spectra is $50 \mathrm{pm}$ in the temperature range from $10^{\circ} \mathrm{C}$ to $60^{\circ} \mathrm{C}$. This result indicates that, as predicted, the designed SMS filter has a wavelength sensitivity similar to that of the FBG.

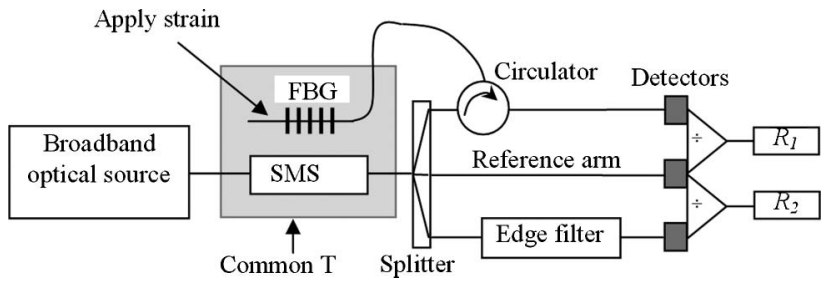

Fig. 9. Schematic diagram of the temperature compensated interrogation system for simultaneous measurements of strain and temperature.
Experiments were carried out by applying strain to the FBG at room temperature $\left(20^{\circ} \mathrm{C}\right)$. The measured ratio versus strain (bottom axis) is shown in Fig. 7 .

To investigate the influence of temperature on the system ratio variations, experiments were carried out by increasing temperature from 10 to $60^{\circ} \mathrm{C}$ for three fixed strain values: 0,667 , and $1667 \mu \varepsilon$. The measured ratio variations versus temperature (top axis) are also shown in Fig. 7.

From Fig. 7 one can see that in the temperature range from $1 \overline{0}$ to $60^{\circ} \mathrm{C}$ the ratio variations are small for all three fixed strain values. Our calculation shows that the maximum strain variation (and hence error) is $34 \mu \varepsilon$. This result could be further improved if the SMS filter used for temperature compensation has a temperature sensitivity closer to that of the FBG in use.

Figure 7 also shows that as the strain increases from 0 to $1667 \mu \varepsilon$, the measured ratio decreases in absolute value monotonically. To illustrate the resolution of this system, incremental step changes of $67 \mu \varepsilon$ and $6.7 \mu \varepsilon$ are applied to the FBG sensor for

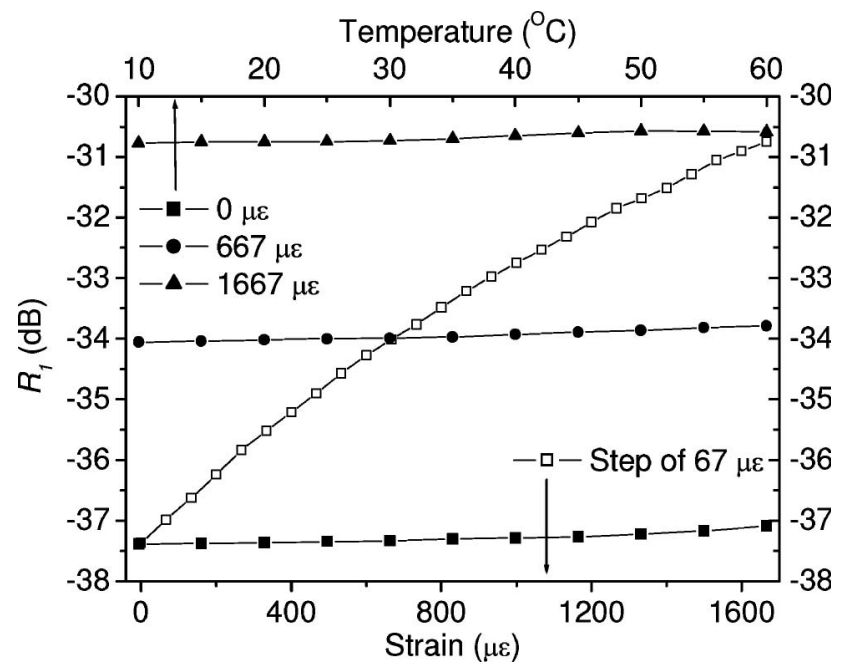

Fig. 10. Measured ratio $R_{1}$ versus strain at room temperature (bottom axis) and measured ratio versus temperature at fixed strain of 0,667 , and $1667 \mu \varepsilon$ (top axis). 

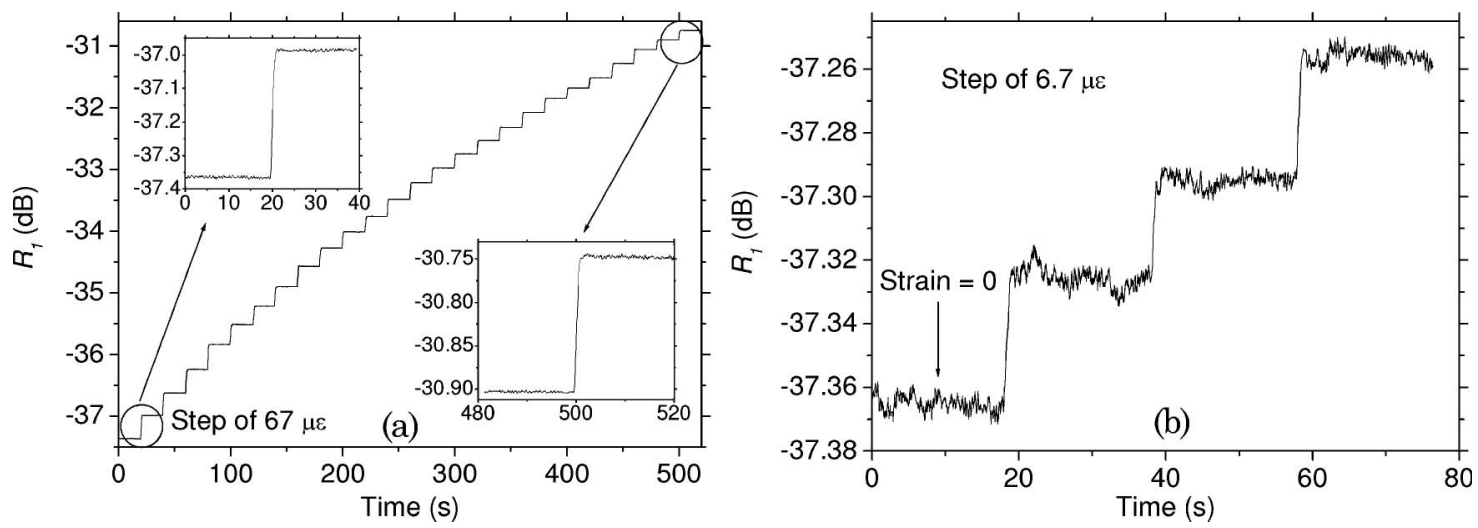

Fig. 11. Measured $R_{1}$ ratio variations versus time with step change of (a) $67 \mu \varepsilon$ from 0 to $1667 \mu \varepsilon$ and (b) $6.7 \mu \varepsilon$ from 0 to $20 \mu \varepsilon$.

comparison. The corresponding measured ratio variations versus time are shown in Figs. 8(a) and 8(b).

It should also be noted that the dependence of the measured ratio versus applied strain deviates from linear (see Fig. 7). The result of this nonlinearity can be seen in Fig. 8(a) where, as the applied strain increases, the ratio variation rate decreases, reaching a minimum value in the strain range from 1600 to $1667 \mu \varepsilon$. Figure 8(b) illustrates small strain step changes of $6.7 \mu \varepsilon$ from 0 to $20 \mu \varepsilon$.

For a ratiometric system, we assume that a minimum reliable detectable ratio variation is equal to the peak-to-peak ratio fluctuation $\Delta R_{p-p}$. If the slope of the ratio response of a system is $m$, then the system resolution will be equal to $\Delta R_{p-p} / \mathrm{m}$. Figures $\underline{7}$ and 8(a) show that there is a maximum and a minimum slope, and hence the best resolution of $1.7 \mu \varepsilon$ is achieved in the strain range from 0 to $20 \mu \varepsilon$, and the worst resolution of $3.4 \mu \varepsilon$ is achieved in the strain range from 1600 to $1667 \mu \varepsilon$.

\section{Adaptation of the System to Measure Temperature and Strain Simultaneously}

The system presented in Fig. 1 can be used for measurement of strain with dynamic temperature compensation but cannot be used for simultaneous measurements of temperature and strain. In order to measure both strain and temperature simulta-

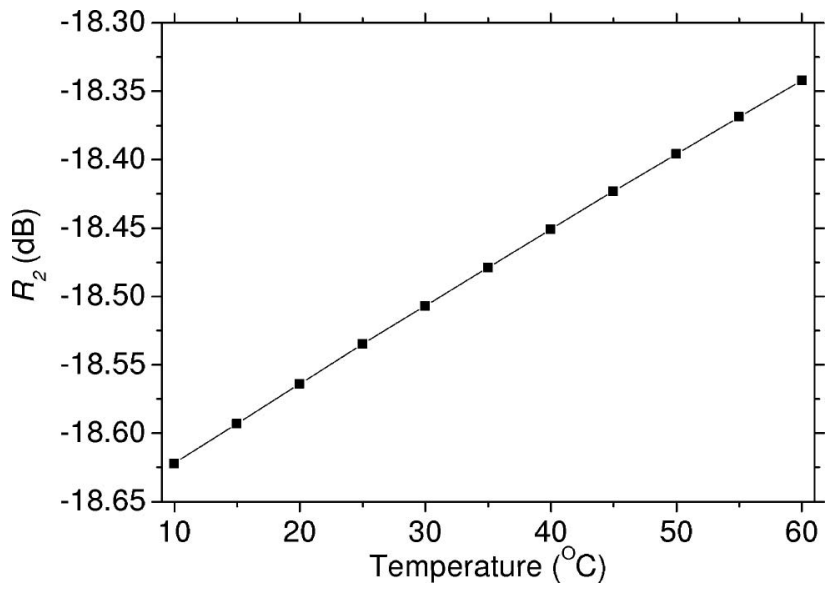

Fig. 12. Measured ratio $R_{2}$ versus temperature. neously, an expanded system configuration is proposed as shown in Fig. 9 .

The light from a broadband optical source passes through an SMS filter and then is split into three paths: one is directed to the FBG, and the reflected signal is detected by a photodiode; another part of the signal is directly applied to a photodiode as a reference; the last part passes through an edge filter and is also detected by a photodiode. Both the FBG and the SMS filter are at the same temperature (in the experiment both devices were collocated in the same heating unit), but strain is applied to the FBG sensor only. Since the SMS filter is only influenced by the temperature, temperature information can be extracted by monitoring the ratio $R_{2}$. If both the FBG and SMS filter have similar temperature sensitivities and the input broadband optical source is spectrally flat, the ratio $R_{1}$ will only reflect strain variations. Hence a system with such configuration can be used to measure both strain and temperature simultaneously with dynamic temperature compensation of strain measurement.

Experiments were carried out to investigate the properties of the system proposed in Fig. 9. The FBG sensor and SMS filter used are the same as described in Fig. 5. The second edge filter used is a thin-film filter.

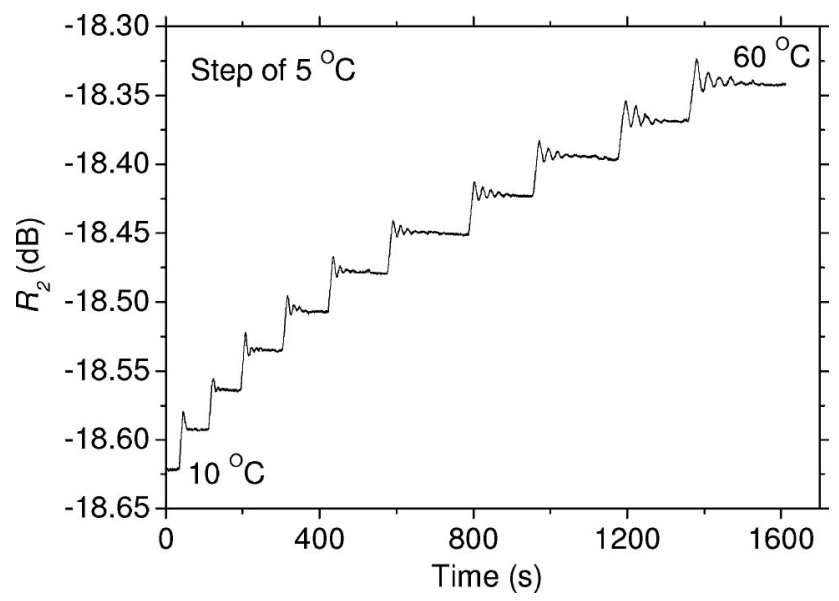

Fig. 13. $R_{2}$ versus time with step changes of $5{ }^{\circ} \mathrm{C}$. 

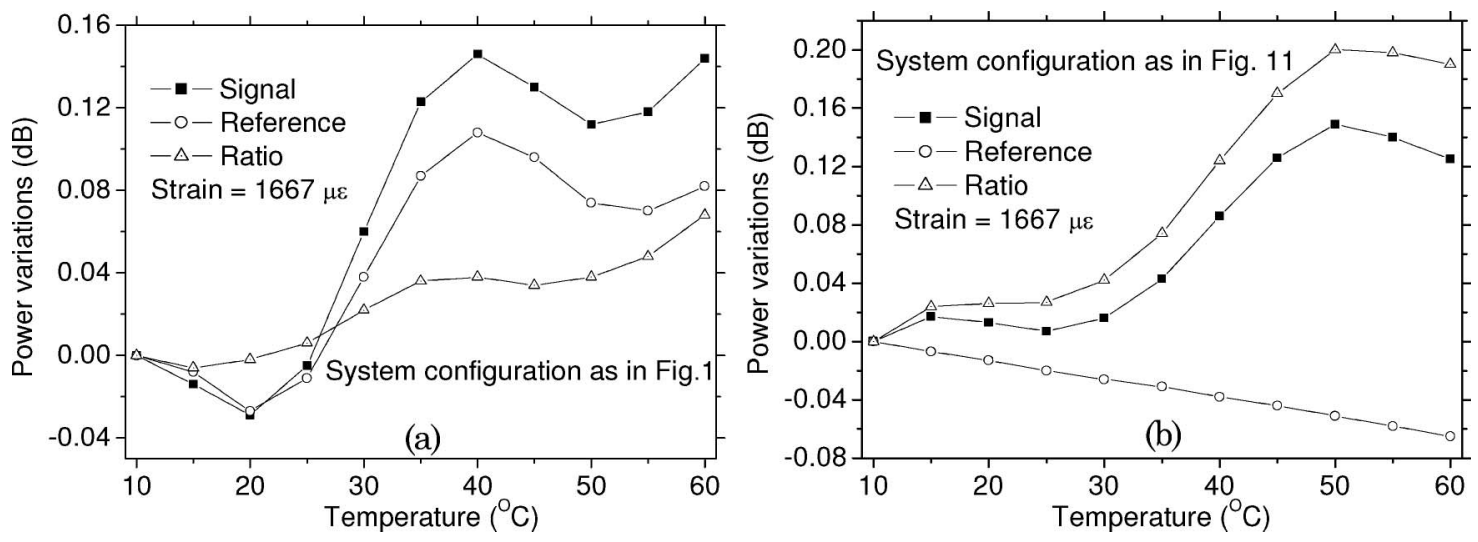

Fig. 14. Measured signal, reference, and $R_{2}$ ratio variations versus temperature for system configurations as in (a) Fig. 1 and in (b) Fig. $\underline{9}$.

The measured ratio versus strain (bottom axis) at room temperature $\left(20^{\circ} \mathrm{C}\right)$ is shown in Fig. 10 along with the dependencies of the ratio versus temperature (top axis) for three different strain values $(0$, 667 , and $1667 \mu \varepsilon$ ).

It can be calculated that the temperature induced ratio variation in the range from 10 to $60^{\circ} \mathrm{C}$ is equal to $82 \mu \varepsilon$, which is 2.4 times that in Fig. 7 .

Figure 11 presents the measured $R_{1}$ ratio variations versus time with incremental step changes of $67 \mu \varepsilon$ and $6.7 \mu \varepsilon$.

Figure 11 shows that the ratio response is similar to that in Fig. 8 . Our calculation shows that the minimum resolution of the interrogation system is $4.3 \mu \varepsilon$.

The variation in the ratio $R_{2}$ versus temperature was measured and is shown in Fig. 12.

As one can see from Fig. 12, the ratio $R_{2}$ is a linear function of temperature with a discrimination of $0.28 \mathrm{~dB}$ in the range from 10 to $60^{\circ} \mathrm{C}$. Figure 13 illustrates the $R_{2}$ ratio variations versus time when temperature changes from 10 to $60^{\circ} \mathrm{C}$ by incremental steps of $5{ }^{\circ} \mathrm{C}$ are made.

The maximum ratio fluctuation at a constant temperature is $0.005 \mathrm{~dB}$, and the minimum ratio variation is $0.027 \mathrm{~dB}$ from 55 to $60^{\circ} \mathrm{C}$. Hence the minimum resolution for temperature is $1^{\circ} \mathrm{C}$.

The system with the configuration shown in Fig. 9 can be used to simultaneously measure strain and temperature as described above; however, the temperature induced error is higher than that for the system in Fig. 1. Our detailed investigation shows that in both system configurations, the power variations versus temperature are similar at the circulator signal arm, but are quite different at the reference arm. Figure 14 presents measured results for both system configurations at a fixed strain of $1667 \mu \varepsilon$.

From Figs. 14(a) and 14(b) one can see that temperature induced power variations at the circulator signal arm for both systems are similar and reach a maximum of circa $0.15 \mathrm{~dB}$; however, the power fluctuations at the reference arm are significantly different: for the system configuration in Fig. 1 the temperature induced power variations at the reference arm are similar to those at the circulator signal arm, resulting in relatively small ratio variations, while for the system configuration in Fig. $\underline{9}$, the nature of the temperature dependence of the power at the reference arm results in large temperature induced ratio variations. One possible reason for such a difference in temperature induced power variations at the two reference arms is the nonflatness of the output spectrum of the broadband optical source.

\section{Conclusions}

In this paper we have proposed a ratiometric interrogation technique with dynamic temperature compensation for FBG strain sensors. This technique uses a SMS fiber filter as a temperature compensating element. We developed a design process for creating SMS edge filters with a temperature sensitivity similar to that of a typical FBG $\left(10 \mathrm{pm} /{ }^{\circ} \mathrm{C}\right)$ based on optimization of the properties of the MMF section. The use of the proposed technique for interrogation of an FBG strain sensor is also experimentally demonstrated. Experimental results show that the proposed technique offers a resolution better than $3.4 \mu \varepsilon$ in the range of strains from 0 to $1667 \mu \varepsilon$ and a temperature induced error as low as $34 \mu \varepsilon$ in the temperature range from 10 to $60^{\circ} \mathrm{C}$. Since the temperature sensitivity of the SMS filter used in our experiments differed slightly from that of the FBG sensor (there was a relative wavelength shift of $50 \mathrm{pm}$ between the spectral responses of the FBG and the SMS edge filter over the temperature range from 10 to $60^{\circ} \mathrm{C}$ ), the temperature induced error can be further reduced by choosing a MMF with the required TOC and TEC. Additionally we demonstrated the possibility of simultaneous measurements of temperature and strain by using a modified system configuration that still incorporated dynamic temperature compensation of strain measurement. A temperature resolution better than $1{ }^{\circ} \mathrm{C}$ was achieved in our experiment and can be improved by using a higher slope edge filter if required.

\section{References}

1. Y. B. Lin, C. L. Pan, Y. H. Kuo, K. C. Chang, and J. C. Chern, "Online monitoring of highway bridge construction using fiber Bragg grating sensors," Smart Mater. Struct. 14, 1075-1082 (2005). 
2. A. Hongo, S. Kojima, and S. Komatsuzaki, "Applications of fiber Bragg grating sensors and high-speed interrogation techniques," Struct. Control Health Monitor. 12, 269-282 (2005).

3. G. P. Brady, K. Kalli, D. J. Webb, D. A. Jackson, L. Reekie, and J. L. Archambault, "Simultaneous measurement of strain and temperature using the first- and second-order diffraction wavelengths of Bragg gratings," IEE Proc. Optoelectron. 144, 156-161(1997).

4. F. M. Hanran, J. K. Rew, and P. D. Foote, "A strain-isolated fibre Bragg grating sensor for temperature compensation of fibre Bragg grating strain sensors," Meas. Sci. Technol. 9, 1163-1166 (1998).

5. L. Lin, W. G. Zhang, H. Zhang, B. Liu, H. Zhao, Q. C. Tu, G. Y. Kai, and X. Y. Dong, "An embedded FBG sensor for simultaneous measurement of stress and temperature," IEEE Photon. Technol. Lett. 18, 154-156 (2006).

6. L. Y. Shao, X. Y. Dong, A. P. Zhang, H. Y. Tam, and S. L. He, "High-resolution strain and temperature sensor based on distributed Bragg reflector fiber laser," IEEE Photon. Technol. Lett. 19, 1598-1600 (2007).

7. L. V. Nguyen, D. Hwang, D. S. Moon, and Y. Chung, "Simultaneous measurement of temperature and strain using a Lyot fiber filter incorporated with a fiber Bragg grating in a linear configuration," Meas. Sci. Technol. 20, 034006 (2009).

8. S. M. Melle, K. Liu, and R. M. Measures, "A passive wavelength demodulation system for guided-wave Bragg grating sensors," IEEE Photon. Technol. Lett. 4, 516-518 (1992).

9. G. Dooly, C. Fitzpatrick, and E. Lewis, "Deep UV based DOAS system for the monitoring of nitric oxide using ratiometric separation techniques," Sens. Actuators B 134, 317-323 (2008).

10. Y. P. Miao, B. Liu, W. H. Zhang, B. Dong, H. B. Zhou, and Q. D. Zhao, "Dynamic temperature compensating interrogation technique for strain sensors with tilted fiber Bragg gratings," IEEE Photon. Technol. Lett. 20, 1393-1395 (2008).

11. D. P. Zhou, L. Wei, W. K. Liu, and J. W. Y. Lit, "Simultaneous strain and temperature measurement with fiber Bragg grating and multimode fibers using an intensity-based interrogation method," IEEE Photon. Technol. Lett. 21, 468-470 (2009).

12. Q. Wang, G. Farrell, and W. Yan, "Investigation on singlemodemultimode- singlemode fiber structure," J. Lightwave Technol. 26, 512-519 (2008).

13. W. S. Mohammed, P. W. E. Smith, and X. Gu, "All-fiber multimode interference bandpass filter," Opt. Lett. 31, 2547-2549 (2006).

14. A. M. Hatta, G. Farrell, Q. Wang, G. Rajan, P. Wang, and Y. Semenova, "Ratiometric wavelength monitor based on singlemode-multimode-singlemode fiber structure," Microwave Opt. Technol. Lett. 50, 3036-3039 (2008).

15. E. Li, "Temperature compensation of multimode interferencebased fiber devices," Opt. Lett. 32, 2064-2066 (2007). 